\title{
RESPUESTA DE LA VERDOLAGA (Portulaca oleracea L.) A LA FERTILIZACIÓN CON NPK
}

\author{
PURSLANE (Portulaca oleracea L.) RESPONSE TO NPK FERTILIZATION
}

\author{
César O. Montoya-García ${ }^{1 *}$, Víctor Volke-Haller ${ }^{1}$, Antonio Trinidad-Santos', \\ Clemente Villanueva-Verduzco² y Julio Sánchez-Escudero ${ }^{1}$
}

\begin{abstract}
'Colegio de Postgraduados, Campus Montecillo. km 36.5 Carretera México-Texcoco, Montecillo, Texcoco. 56230, Estado de México. ${ }^{2}$ Departamento de Fitotecnia, Universidad Autónoma Chapingo. km 38.5 Carretera México-Texcoco, Chapingo, Texcoco. 56230, Estado de México.
\end{abstract}

*Autor para correspondencia (montoya.cesar@colpos.mx)

\section{RESUMEN}

La producción de verdolaga (Portulaca oleracea L.) en México se realiza en condiciones de campo y no existen estudios sobre dosis de fertilización de NPK que maximicen el rendimiento del follaje. El objetivo del estudio fue evaluar el efecto de la fertilización con N, Py K en la producción de verdolaga, así como determinar la dosis óptima económica. El estudio se realizó en condiciones de campo y se aplicaron cuatro dosis de $\mathrm{N}\left(0,100,200\right.$ y $\left.300 \mathrm{~kg} \mathrm{~N} \mathrm{ha}^{-1}\right), \mathrm{P}(0$, 30,60 y $\left.90 \mathrm{~kg} \mathrm{P}_{2} \mathrm{O}_{5} \mathrm{ha}^{-1}\right)$ y $\mathrm{K}\left(0,40,80\right.$ y $\left.120 \mathrm{~kg} \mathrm{~K}_{2} \mathrm{O} \mathrm{ha}{ }^{-1}\right)$, en un diseño de bloques completos al azar con cuatro repeticiones. Las variables evaluadas fueron altura de planta, concentración de $\mathrm{N}$ y nitratos, y rendimiento en tres fechas de cosecha comercial $(27,34$ y 42, días después de la emergencia). La altura y rendimiento se incrementaron por el $\mathrm{N}$ aplicado y la fecha de cosecha. Se observó variación en la germinación de la semilla con densidades de plantas de 1100 a 2500 plantas $\mathrm{m}^{-2}$ que afectó el rendimiento. No se observó efecto por el P y K aplicados. El mayor rendimiento fue con $100 \mathrm{~kg} \mathrm{~N} \mathrm{ha}^{-1} \mathrm{y}$ 2500 plantas $\mathrm{m}^{-2}$ en la primera, segunda y tercera cosecha $(60,97$ y $132 \mathrm{t}$ $\mathrm{ha}^{-1}$, respectivamente). El N y P aplicados incrementaron la concentración de $\mathrm{N}$ y nitratos; sin embargo, ésta no es mayor que la cantidad permitida para su consumo en fresco. La dosis óptima económica de $\mathrm{N}$ se estimó en $65 \mathrm{~kg} \mathrm{~N} \mathrm{ha}^{-1}$ y 2500 plantas $\mathrm{m}^{-2}$ en las tres cosechas, con rendimientos del follaje de 58, 95 y $130 \mathrm{t} \mathrm{ha}^{-1}$, e ingresos netos de 30338, 67828 y 102440 pesos mexicanos por hectárea, para las tres cosechas, respectivamente. Los resultados indican la dosis de $\mathrm{N}$ que maximiza el rendimiento del follaje y los beneficios económicos.

Palabas clave: Portulaca oleracea, dosis óptima económica, concentración de nitratos, concentración de nitrógeno, fertilización nitrogenada.

\section{SUMMARY}

Purslane (Portulaca oleracea L.) production in Mexico is carried out under field conditions and there are no studies on NPK fertilization doses that maximize foliage yield. The objective of the study was to evaluate the effect of fertilization with $\mathrm{N}, \mathrm{P}$ and $\mathrm{K}$ over purslane production, as well as to determine the optimal economic dose. The study was performed under field conditions and four doses of $\mathrm{N}\left(0,100,200\right.$ and $\left.300 \mathrm{~kg} \mathrm{~N} \mathrm{ha}^{-1}\right), \mathrm{P}(0,30,60$ and $90 \mathrm{~kg}$ $\mathrm{P}_{2} \mathrm{O}_{5}$ ha $\left.^{-1}\right)$ and $\mathrm{K}\left(0,40,80\right.$ and $120 \mathrm{~kg} \mathrm{P}_{2} \mathrm{O}_{5}$ ha- $\left.^{-1}\right)$, were applied in a randomized complete blocks design with four replications. Traits evaluated were plant height, $\mathrm{N}$ and nitrates concentration and yield on three commercial harvest dates (27, 34 y 42 days after emergence). Height and yield increased by the applied $\mathrm{N}$ and the harvest date. Variation was observed on seed germination with plant densities of 1100 to 2500 plants $\mathrm{m}^{-2}$ that affected the yield. No effect was observed for the applied $\mathrm{P}$ and $\mathrm{K}$. The greatest yield was with 100 $\mathrm{kg} \mathrm{N} \mathrm{ha}^{-1}$ and 2500 plants $\mathrm{m}^{-2}$ at the first, second and third harvests $(60,97$ and $132 \mathrm{tha}^{-1}$, respectively). The applied $\mathrm{N}$ and $\mathrm{P}$ increased the concentration of $\mathrm{N}$ and nitrates, however, this is not greater than the amount allowed for fresh consumption. The economical optimal dose of $\mathrm{N}$ was estimated at $65 \mathrm{~kg}$ $\mathrm{N} \mathrm{ha}^{-1}$ and 2500 plants $\mathrm{m}^{-2}$ in the three harvests, with foliage yields of 58,95 and $130 \mathrm{t} \mathrm{ha}^{-1}$, and net income of 30338, 67828 and 102440 Mexican pesos per hectare, for the three harvests, respectively. Results indicate the dose of $\mathrm{N}$ that maximizes foliage yield and economic benefits.

Index words: Portulaca oleracea, economical optimal doses, nitrates concentration, nitrogen concentration, nitrogen fertilization.

\section{INTRODUCCIÓN}

La verdolaga (Portulaca oleracea L.) es una de las ocho plantas más comunes en el mundo (Liu et al., 2000), y a pesar de que su origen está en discusión, ya existía en el continente americano antes de la llegada de Cristóbal Colón (Byrne y McAndrews, 1975; Chapman et al., 1973). En el Códice Florentino se describe a la verdolaga como una planta de tallo grueso, hojas pequeñas y redondas y de hábito rastrero (Dibble y Anderson, 1963). Los estudios arqueobotánicos realizados en la Cuenca de México revelan la presencia de la verdolaga como planta comestible desde el periodo Preclásico, al sur de esta cuenca (McClung de Tapia et al., 2014), y en el periodo postclásico temprano en la zona de Teotihuacán, Xaltocan e Iztapalapa (Brumfiel, 2009; McClung de Tapia et al., 2014; Vázquez-Alonso et al., 2014).

La verdolaga ofrece una alternativa como cultivo hortícola por su contenido de ácidos grasos (a-linolénico y glinolénico), proteínas, aminoácidos y antioxidantes como a-tocoferol, vitamina C, B-caroteno, polifenoles y flavonoides (Liu et al., 2000; Palaniswamy et al., 2000; Siriamornpun y Suttajit, 2010).

El cultivo comercial de la verdolaga en México se inició en 1950 en la zona chinampera de la Ciudad de México (López, 1996); actualmente se utilizan variedades propias de la región, domesticadas por los productores, y se siembra una superficie de 454 ha en condiciones de riego y temporal. En los estados de Morelos, Baja California y 
la Ciudad de México se obtienen rendimientos medios de 19.1, 10.1 y 9.4 t ha $^{-1}$, respectivamente; sin embargo, los productores de la Ciudad de México mencionan que pueden obtener rendimientos máximos de 30 a 40 t ha $^{-1}$.

Los productores de verdolaga de la zona de Mixquic, Ciudad de México suelen fertilizar con 14 t de estiércol de bovino o $3 \mathrm{t}$ de gallinaza por hectárea o ambas enmiendas; sin embargo, con la intención de hacer más eficiente la fertilización para alcanzar rendimientos óptimos, evitar contaminación del suelo y acumulación de nitratos en el cultivo, y racionalizar los costos de producción, es necesario determinar la respuesta de la verdolaga a la fertilización inorgánica.

Los objetivos de la presente investigación fueron: 1) cuantificar el efecto de la fertilización con N, P y K en la producción de la verdolaga; 2) determinar las dosis óptimas económicas de N, P y K para la producción de verdolaga; y, 3) determinar la concentración de $\mathrm{N}$ y nitratos en la verdolaga.

\section{MATERIALES Y MÉTODOS}

\section{Ubicación del estudio}

El estudio se llevó a cabo en el Campo Experimental del Colegio de Postgraduados, Campus Montecillo, Estado de México (19²8' 2.07" N y 98 54' 1.71" O, 2215 msnm), en condiciones de campo, en los meses de julio y agosto de 2014. De acuerdo con García (1973), el clima de la zona es templado Bs 1 kwe (w) (i') g, con una precipitación media anual de $625 \mathrm{~mm}$ y un régimen de lluvias en los meses de julio a septiembre y temperatura media anual de $16^{\circ} \mathrm{C}$. El suelo es clasificado como Mollic Ustifluvent (Soil Survey Staff, 2010), con una profundidad de $150 \mathrm{~cm}$ y una textura franco arcillo limosa en el horizonte Ap.

\section{Precipitación y temperatura durante el ciclo de cultivo}

En la Figura 1 se presentan los datos de las temperaturas máximas y mínimas diarias durante el desarrollo del cultivo de la verdolaga en los meses de julio y agosto de 2014, obtenidos de la Estación Agroclimática de Colegio de Postgraduados, Campus Montecillo, Estado de México. Las precipitaciones ocurridas en los meses de junio, julio y agosto de 2014 fueron de 135.2, 142.5 y $134.1 \mathrm{~mm}$, respectivamente. Las temperaturas máximas medias diarias variaron entre 19 y $30^{\circ} \mathrm{C}$ en los meses de julio y agosto, a la vez que las temperaturas mínimas medias diarias disminuyeron de 11 a $6{ }^{\circ} \mathrm{C}$, durante el desarrollo del cultivo.

\section{Material vegetal}

El material vegetal se obtuvo del pueblo de Mixquic, Tláhuac, Ciudad de México, que se caracteriza por tener un hábito de crecimiento erecto y poco ramificado, hojas grandes y tallos de color verde.

\section{Tratamientos, diseño y unidad experimental}

Se utilizaron cuatro niveles de N, P y K: 0, 100, 200 y 300 $\mathrm{kg} \mathrm{N} \mathrm{ha}^{-1} ;$ 0, 30, 60 y $90 \mathrm{~kg} \mathrm{P}_{2} \mathrm{O}_{5}$ ha-1 $^{-1}$ y 90, 40, 80 y $120 \mathrm{~kg}$ $\mathrm{K}_{2} \mathrm{O} \mathrm{ha}^{-1}$, dando como resultado 12 tratamientos. Las fuentes de fertilizantes fueron sulfato de amonio $\left[\left(\mathrm{NH}_{4}\right)_{2} \mathrm{SO}_{4}\right.$ $21 \% \mathrm{~N}$, fosfato mono-amónico $\left[\mathrm{NH}_{4} \mathrm{H}_{2} \mathrm{PO}_{4}, 12 \% \mathrm{~N}\right.$ y 61 $\left.\% \mathrm{P}_{2} \mathrm{O}_{5}\right]$ y sulfato de potasio $\left[\mathrm{K}_{2} \mathrm{SO}_{4}, 51 \% \mathrm{~K}_{2} \mathrm{O}\right]$. Se utilizó un diseño experimental de bloques completos al azar con cuatro repeticiones. Los niveles de cada elemento se definieron con base en información de la literatura acerca de la extracción nutrimental que hace la planta, en función de

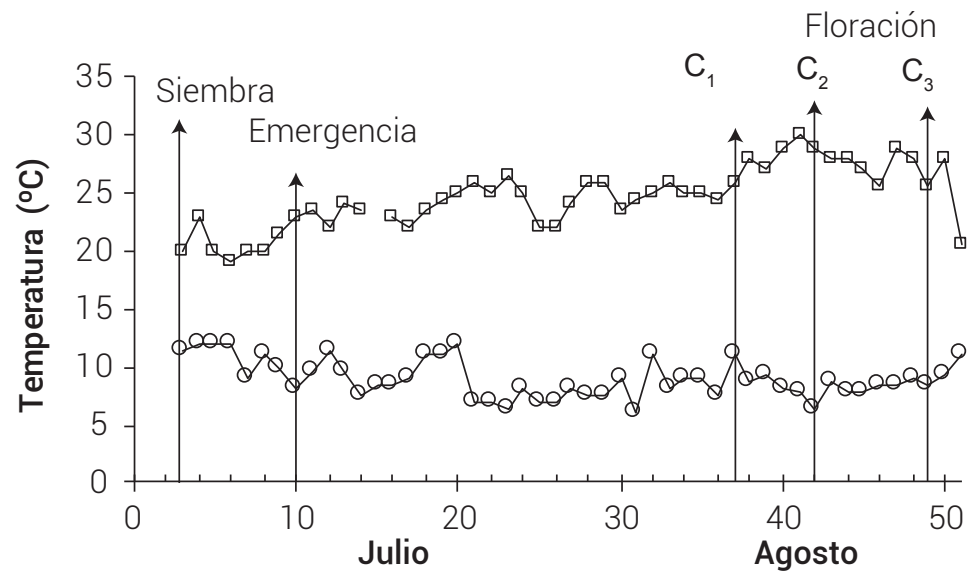

Figura 1. Temperaturas máximas y temperaturas mínimas durante el desarrollo del cultivo de la verdolaga (Portulaca oleracea L.). $\mathrm{C}_{1}$ : cosecha 1 (27 dde); $\mathrm{C}_{2}$ : cosecha 2 (34 dde); $\mathrm{C}_{3}$ : cosecha 3 (42 dde). 
un rendimiento dado y la concentración del elemento. Las unidades experimentales consistieron en melgas de $3 \mathrm{~m}$ de largo por $1.5 \mathrm{~m}$ de ancho.

\section{Conducción del experimento}

La preparación del suelo y siembra se realizaron de manera tradicional, de acuerdo con los procedimientos de los campesinos de Mixquic, que consiste en un barbecho y rastreo, la formación de canales o "regaderas" y las "melgas", y enseguida se da contorno a la melga con bordos de $0.30 \mathrm{~m}$ de ancho. La siembra se realizó el 3 de julio de 2014, esparciendo al voleo $1.86 \mathrm{~g}$ de semilla $\mathrm{m}^{-2}$ (4726 semillas $\mathrm{g}^{-1}$ ). De la misma manera se aplicaron $3.5 \mathrm{t} \mathrm{ha}^{-1}$ de gallinaza, con la finalidad de estimular la germinación. La emergencia se presentó 7 días después de la siembra (dds). A los 25 dds se aplicó al voleo la mitad del fertilizante nitrogenado y todo el P y K de cada uno de los niveles, y el resto del $\mathrm{N}$ a los $32 \mathrm{dds}$. El control de malezas se realizó de manera manual. No fue necesaria la aplicación de insecticidas ni fungicidas. Se realizaron tres cosechas: a madurez vegetativa, 27 días después de la emergencia $\left(C_{1}\right), 34$ días después de la emergencia (primera floración) $\left(C_{2}\right)$ y 42 días después de la emergencia (segunda floración) $\left(\mathrm{C}_{3}\right)$.

\section{Muestreo y análisis de suelos}

En cada unidad experimental se tomaron 10 submuestras de 0 a $20 \mathrm{~cm}$ de profundidad, para obtener una muestra compuesta. Para cada repetición se determinó pH y conductividad eléctrica (CE) (Richards, 1990); bases intercambiables (Ca, Mg y Na) (Chapman, 1965); Fe, Cu, Zn y Mn (Jackson, 1976); y textura (Bouyoucos, 1936). Para cada sub-experimento de N, P y K, por tratamiento y repetición, se determinó materia orgánica (Jackson, 1976); P-Olsen (Olsen y Dean, 1965); y K intercambiable (Chapman, 1965). Los valores obtenidos en el análisis del sitio experimental fueron pH 8.2, CE 0.36 dS m-1, materia orgánica 2.29-3.0\%, P 12-35 mg kg-1, K 546-819 mg kg-1, Ca $560-780 \mathrm{mg} \mathrm{kg}^{-1}$, Mg 125-192 mg kg-1, Na $6.9 \mathrm{mg} \mathrm{kg}^{-1}$, Fe $9 \mathrm{mg} \mathrm{kg}^{-1}$, Cu 1.3 $\mathrm{mg} \mathrm{kg}^{-1}$, Zn 24-29 mg kg-1, Mn $1.5 \mathrm{mg} \mathrm{kg}^{-1}$. La textura fue clasificada como franco arcillo limosa.

\section{Variables de estudio}

Las variables se evaluaron en un área delimitada por un marco de $0.0625 \mathrm{~m}^{2}$, colocado al azar en cada unidad experimental. En las tres fechas de cosecha se tomaron muestras de follaje, se contó el número de plantas, se midió la altura media, se obtuvo el peso fresco y la concentración de nitratos en fresco (Coltman, 1989). Las muestras se secaron en estufa a $70^{\circ} \mathrm{C}$ durante $72 \mathrm{~h}$ para determinar la concentración de N (Alcántar y Sandoval, 1999). La concentración de proteína se obtuvo multiplicando la concen- tración de N por el factor 6.25 (Fontana et al., 2006).

\section{Análisis estadístico de la información}

Para cada variable se estimó un modelo de regresión en función de N, P y K aplicados, que incluyeron a la materia orgánica, P-Olsen y K-intercambiable, en cada sub-experimento, respectivamente. Las fechas de cosecha fueron variables auxiliares, donde la primera fue la base de comparación. Para estimar el modelo de regresión se siguió el procedimiento descrito por Volke (2008).

Con el modelo estimado se calcularon los valores de altura y rendimiento de follaje para los niveles de N, P y K en cada fecha de cosecha, para la representación gráfica de la respuesta, al igual que para la concentración de $\mathrm{N}$ y de nitratos en el follaje.

\section{Recomendaciones de fertilización N, P y K}

La determinación de las dosis óptimas económicas de N, P y K se hizo a partir de los modelos de regresión obtenidos para el rendimiento. Para esto se partió de la siguiente función de ingresos netos: $I N=Y * P y-\left(C F+\sum X i P i\right)$; donde $Y=$ función de producción (modelo de regresión estimado) y Py $=$ precio neto de venta del producto (precio de mercado menos los costos de cosecha y de transporte); CF = costos fijos de producción; $y, \sum X i P i=$ costo variable de los insumos de producción, igual a la cantidad de insumo multiplicado por su costo total, igual a su vez al precio de mercado de la unidad del mismo más el costo por transporte, aplicación y costo del capital. El precio neto de venta de producto se calculó en 1010 pesos por tonelada.

Para calcular el costo de una planta, ya sea que la variable densidad sea considerada como costo fijo o como costo variable, se consideró que en un gramo de semillas existen 4726 semillas, con una germinación de 20 \% (dato obtenido del propio experimento), y un costo de 300 pesos por kilogramo, lo que arroja un costo de 0.318 centavos/ planta; para N, el costo variable se calculó en 29.2 pesos por kilogramo, para P, en 24.2 pesos por kilogramo y para $\mathrm{K}$, en 39.7 pesos por kilogramo. Los costos fijos se calcularon en 17,330 pesos por hectárea. En el cálculo de las dosis óptimas económicas, para todos los insumos, se consideró una tasa de retorno del $100 \%$.

Con base en el criterio de maximización del ingreso neto por superficie, la cantidad óptima económica del mismo se obtuvo derivando la función de ingreso neto con respecto a cada insumo, se igualó la primera derivada a la relación de precios insumo/producto y se resolvió el sistema resultante para los insumos; una vez obtenidas las dosis óptimas económicas para los insumos se obtuvo 
el rendimiento óptimo económico, y se reemplazaron sus cantidades en la función de producción y los costos variables de la función de ingresos netos. Adicionalmente, se calculó la relación beneficio/costo, igual a la relación de los ingresos netos entre los costos totales (Volke, 1982).

\section{RESULTADOS Y DISCUSIÓN}

\section{Altura de planta}

El modelo de regresión para altura de planta en función de los factores en estudio fue el siguiente:

$$
\begin{aligned}
A T= & 17.353+12.0550 C_{2}+19.4604 C_{3}+0.2183 N^{0.50} \\
& \left(\operatorname{Pr} . F=0.0001, C M E=3.134, C V=5.79 \%, R^{2}=0.955\right)
\end{aligned}
$$

donde: $\mathrm{AT}=$ altura de planta $(\mathrm{cm}), \mathrm{N}=$ dosis de $\mathrm{N}\left(\mathrm{kg} \mathrm{N} \mathrm{ha}^{-1}\right)$,

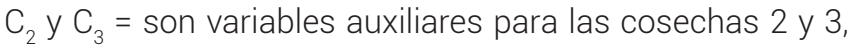
respectivamente, con respecto a la cosecha 1.

La altura de planta se incrementó por el $\mathrm{N}$ aplicado, lo que fue evidente en cada cosecha. La altura se incrementó de $17.3 \mathrm{~cm}$ en $C_{1}$, hasta 29.4 y $36.8 \mathrm{~cm}$ en $\mathrm{C}_{2}$ y $\mathrm{C}_{3}$ con 0 $\mathrm{kg} \mathrm{N} \mathrm{ha}^{-1}$. Por el $\mathrm{N}$ aplicado, se observó respuesta hasta $300 \mathrm{~kg} \mathrm{~N} \mathrm{ha}^{-1}$, dosis máxima probada, con un mayor incremento hasta $100 \mathrm{~kg} \mathrm{~N} \mathrm{ha}^{-1}$ (Figura 2). Por otra parte, no se observó respuesta por el $\mathrm{P}$ y $\mathrm{K}$ aplicados; por lo tanto, el contenido de $\mathrm{P}\left(12 \mathrm{mg} \mathrm{kg}^{-1}\right)$ y K $\left(546 \mathrm{mg} \mathrm{kg}^{-1}\right)$ del suelo cubrió las necesidades del cultivo.

Si bien, son pocos los estudios realizados en verdolaga en campo, Kaymak (2013) obtuvo alturas medias de 23 cm (35 dds) con $150 \mathrm{~kg} \mathrm{~N} \mathrm{ha}^{-1}$, mientras que en sistemas hidropónicos se han reportado alturas máximas de 27.4 y $44.1 \mathrm{~cm}$ a los 18 dds y $36 \mathrm{dds}$, respectivamente (Palaniswamy et al., 2000), de $12.2 \mathrm{~cm}$ a los 50 dds (Cros et al., 2007); y de $12.4 \mathrm{~cm}$ a los 50 dds, con una solución salina a
$2.5 \mathrm{dS} \mathrm{m}^{-1}$ (Franco et al., 2011).

\section{Rendimiento del follaje}

La cantidad de semilla aplicada fue del orden de $1.86 \mathrm{~g}$ $\mathrm{m}^{-2}$, que corresponde a 4726 semillas $\mathrm{m}^{-2}$, lo que quiere decir que para poblaciones de 1100, 1750 y 2500 plantas $\mathrm{m}^{-2}$, la germinación de las semillas fue de 12.5, 19.9 y 28.4 $\%$, respectivamente, valores que son muy bajos. Lo anterior produjo una densidad de plantas por superficie no homogénea, pues ésta fluctuó de 1100 a 2500 plantas $\mathrm{m}^{-2}$, por lo que se incluyó en el siguiente modelo de rendimiento:

$Y=-29.271+37.119 C_{2}+71.388 C_{3}+1.613 D^{0.50}+0.925 N^{0.50}$
$\left(\operatorname{Pr} . F=0.0001, C M E=156.927, C V=15.163, R^{2}=0.844\right)$,

donde: $Y=$ rendimiento de follaje de verdolaga en fresco $(t$ $\left.\mathrm{ha}^{-1}\right), \mathrm{DP}=$ densidad de plantas (plantas $\left.\mathrm{m}^{-2}\right), \mathrm{N}=$ dosis de

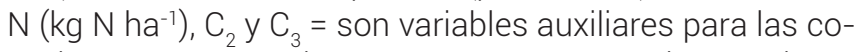
sechas 2 y 3 , respectivamente, con respecto a la cosecha 1 .

El modelo de regresión indica que el rendimiento de follaje se incrementó por la cosecha, el $\mathrm{N}$ aplicado y la densidad de plantas. Por la cosecha hubo un incremento de $37.1 \mathrm{t} \mathrm{ha}^{-1}$ de la cosecha 2 sobre la cosecha 1, y de $34.2 \mathrm{t}$ ha $^{-1}$ de la cosecha 3 sobre la cosecha 2. Por el N aplicado se observó mayor incremento a $100 \mathrm{~kg} \mathrm{~N} \mathrm{ha}^{-1}$, aunque se obtuvo respuesta hasta $300 \mathrm{~kg} \mathrm{~N} \mathrm{ha}^{-1}$, que fue la dosis máxima probada (Figura 3). Por otra parte, el P y K aplicados no causaron efecto sobre el rendimiento de follaje, por lo tanto el suministro de $\mathrm{P}\left(15 \mathrm{mg} \mathrm{kg}^{-1}\right)$ y K $\left(624 \mathrm{mg} \mathrm{kg}^{-1}\right)$ del suelo resultó adecuado para el cultivo.

El mayor rendimiento del follaje debido a la fecha de cosecha indica que las plantas continuaron su crecimiento en el tiempo, y el efecto positivo del $\mathrm{N}$ se deriva generalmente en su importancia sobre el desarrollo de la planta
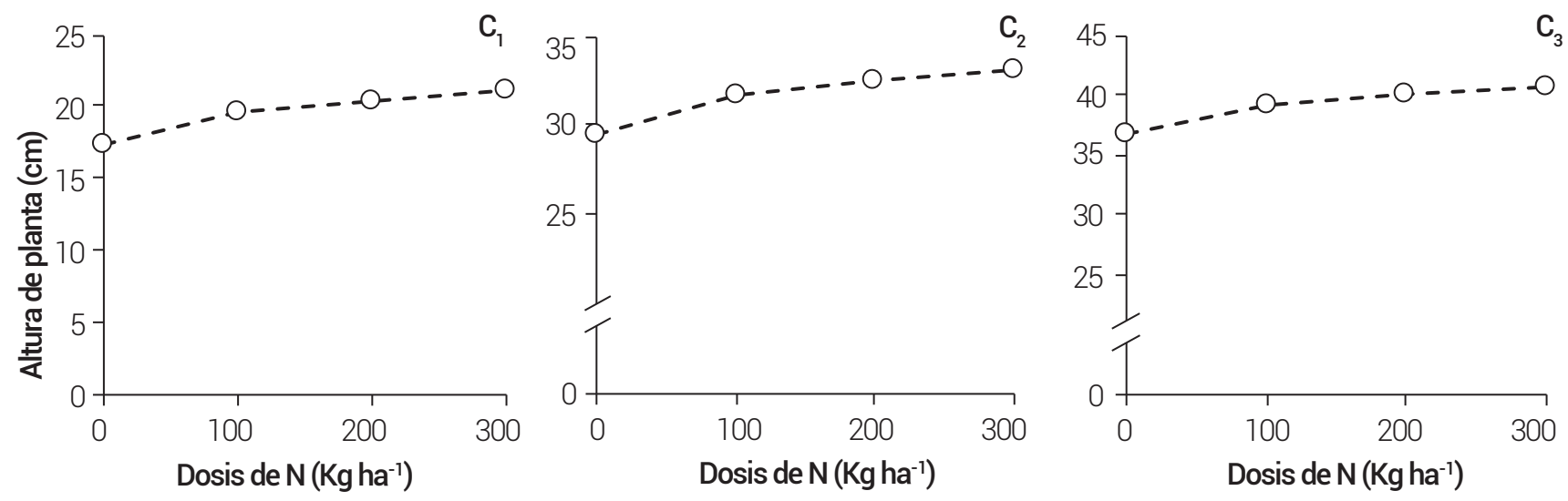

Figura 2. Altura de la verdolaga (Portulaca oleracea L.) en función del $\mathrm{N}$ aplicado y fecha de cosecha: $\mathrm{C}_{1}$ : $\operatorname{cosecha} 1$ a 27 dde; $C_{2}$ : cosecha 2 a 34 dde y $C_{3}$ : cosecha 3 a 42 dde. 

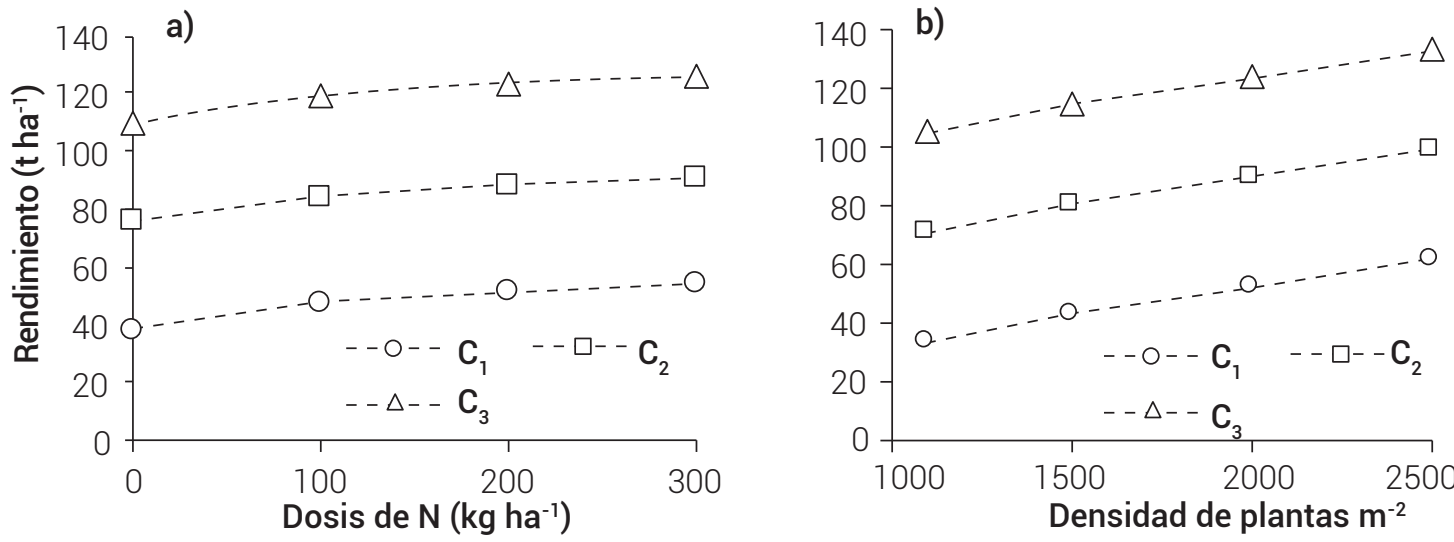

Figura 3. Rendimiento del follaje de la verdolaga (Portulaca oleracea L.) en tres fechas de cosecha. $\mathrm{C}_{1}$ : cosecha 1 a 27 dde; $\mathrm{C}_{2}$ : cosecha 2 a 34 dde y $\mathrm{C}_{3}$ : cosecha 3 a 42 dde. a) Efecto del rendimiento por el $\mathrm{N}$ aplicado con 1750 plantas $\mathrm{m}^{-2}$; b) Efecto del rendimiento por la densidad de plantas $\mathrm{m}^{-2}$ con $100 \mathrm{~kg} \mathrm{~N} \mathrm{ha}^{-1}$.

y que usualmente el suelo no tiene la cantidad suficiente para éste.

El rendimiento del follaje presentó una correlación positiva con la altura de la planta $(r=0.87, P \leq 0.05)$, lo que indica que a mayor altura se espera mayor desarrollo de follaje (hojas y tallos) de la verdolaga.

Comparados con los pocos estudios existentes, los rendimientos aquí obtenidos son altos desde las cosechas tempranas. Petropoulos et al. (2015) reportan rendimientos de $33 \mathrm{t} \mathrm{ha}^{-1}$ a los $65 \mathrm{dds}$, en un cultivar proveniente de Sari, Mazandaran, Irán; Elmi et al. (1997) obtuvieron rendimientos de 37 y $70 \mathrm{t} \mathrm{ha}^{-1}$, en cultivares provenientes de Egipto y Estados Unidos, respectivamente, con $272 \mathrm{~kg} \mathrm{~N}$, $\mathrm{P}_{2} \mathrm{O}_{5}$ y $\mathrm{K}_{2} \mathrm{O} \mathrm{ha}^{-1}$. En sistemas hidropónicos en bandejas flotantes se reportan rendimientos de $2.3 \mathrm{~kg} \mathrm{~m}^{-2}$ a los $20 \mathrm{dds}$ (Fontana et al., 2006), de 1.8 y $3.8 \mathrm{~kg} \mathrm{~m}^{-2}$ a los 18 y $50 \mathrm{dds}$, respectivamente (Cros et al., 2007), de $1.4 \mathrm{~kg} \mathrm{~m}^{-2}$ a los 16 dds (Kaşkar et al., 2009), y de $4 \mathrm{~kg} \mathrm{~m}^{-2}$ a los 16 dds (EgeaGilabert et al., 2014). En condiciones salinas moderadas se ha observado un ligero incremento del rendimiento de 8.7 $\mathrm{kg} \mathrm{m}^{-2}\left(3.5 \mathrm{dS} \mathrm{m}^{-1}\right)$ a los $38 \mathrm{dds}$ (kiliç et al., 2008) y de $3.8 \mathrm{~kg}$ $\mathrm{m}^{-2}$ a los 50 dds (2.5 dS m-1) (Franco et al., 2011).

\section{Concentración de nitrógeno y nitratos en follaje}

Los modelos de regresión para la concentración de $\mathrm{N}$ y nitratos en el follaje en función de los factores de estudio, fueron los siguientes:

$$
\begin{aligned}
& \mathrm{CN}=2.523+0.00520 \mathrm{~N}+0.0003256 \mathrm{~N} \mathrm{P}^{0.50} \\
& \quad\left(\mathrm{Pr} . \mathrm{F}=0.0001, \mathrm{CME}=0.1698, \mathrm{CV}=10.59 \%, \mathrm{R}^{2}=0.642\right), \\
& \mathrm{NO}_{3}{ }^{-}=516.50+3.494 \mathrm{~N}-0.00915 \mathrm{~N}^{2}+0.637 \mathrm{~N} \mathrm{P}^{0.25}+ \\
& 38.992 \mathrm{C}_{2} \mathrm{~N}^{0.50}+89.00947 \mathrm{C}_{3} \mathrm{~N}^{0.25}
\end{aligned}
$$

$$
\left(\operatorname{Pr} . \mathrm{F}=0.0001, \mathrm{CME}=41414, \mathrm{CV}=14.708 \%, \mathrm{R}^{2}=0.741\right)
$$

donde: $\mathrm{CN}$ = concentración de $\mathrm{N}(\%)$ en materia seca, $\mathrm{NO}_{3}{ }^{-}=$ concentración de $\mathrm{NO}_{3}{ }^{-}$en follaje de verdolaga $\left(\mathrm{mg} \mathrm{NO}_{3}{ }^{-} \mathrm{kg}^{-1}\right.$ de materia fresca), $\mathrm{N}=$ dosis de $\mathrm{N}\left(\mathrm{kg} \mathrm{N} \mathrm{ha}^{-1}\right), \mathrm{P}=$ dosis de $\mathrm{P}$ $\left(\mathrm{kg} \mathrm{P}_{2} \mathrm{O}_{5} \mathrm{ha}^{-1}\right) . \mathrm{C}_{2}$ y $\mathrm{C}_{3}=$ son variables auxiliares para la cosecha 2 y 3 , respectivamente, con respecto a la cosecha 1.

El modelo de regresión indica que la concentración de $\mathrm{N}$ se incrementó por el $\mathrm{N}$ y $\mathrm{P}$ aplicados, y una interacción entre el $\mathrm{N}$ y $\mathrm{P}$ aplicados. Para el $\mathrm{N}$ se observó un incremento desde $2.5 \%$ de $\mathrm{N}$ en materia seca (MS), concentración obtenida con $0 \mathrm{~kg} \mathrm{~N}^{-1}$, hasta $4.0 \%$ de $\mathrm{N}$ en MS

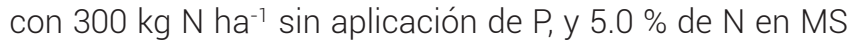

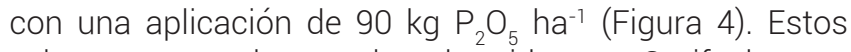
valores concuerdan con los obtenidos por Graifenberg et al. (2003) y Kaymak (2013), que reportaron 2.8 y $2.4 \%$ de $\mathrm{N}$, respectivamente; pero son inferiores a los de Fontana et al. (2006), que reportaron $6.3 \%$ de N en MS. Por otra parte, la concentración de proteína osciló entre 15 y 31 \%. El máximo contenido de proteína en verdolaga es reportado por Mohamed y Hussein (1994) y Fontana et al. (2006), quienes reportaron 44 y $39 \%$ de proteína en MS, respectivamente. La alta salinidad tiene un efecto perjudicial sobre la concentración de proteína en hojas (Teixeira y Carvalho, 2009; Uddin et al., 2012); mientras que la fecha de siembra (primavera o verano) y el cultivar tiene un efecto directo en la concentración de proteína (Ezekewe et al., 1999).

El modelo de regresión indica que la concentración de $\mathrm{NO}_{3}{ }_{3}^{-}$se incrementó por el $\mathrm{N}$ aplicado, y una interacción entre el $\mathrm{N}$ y el $\mathrm{P}$ aplicados, y entre el $\mathrm{N}$ aplicado y la fecha de cosecha. La verdolaga concentra $516 \mathrm{mg} \mathrm{NO}_{3}{ }^{-} \mathrm{kg}^{-1} \mathrm{de}$ materia fresca (MF) sin aplicación de N y P en las tres cosechas, e incrementa hasta valores de 1329, 2005 y 1700 $\mathrm{mg} \mathrm{NO}_{3}{ }^{-} \mathrm{kg}^{-1} \mathrm{MF}$ obtenidas con $300 \mathrm{~kg} \mathrm{~N}^{-1}$ y $90 \mathrm{~kg} \mathrm{P}_{2} \mathrm{O}_{5}$ 


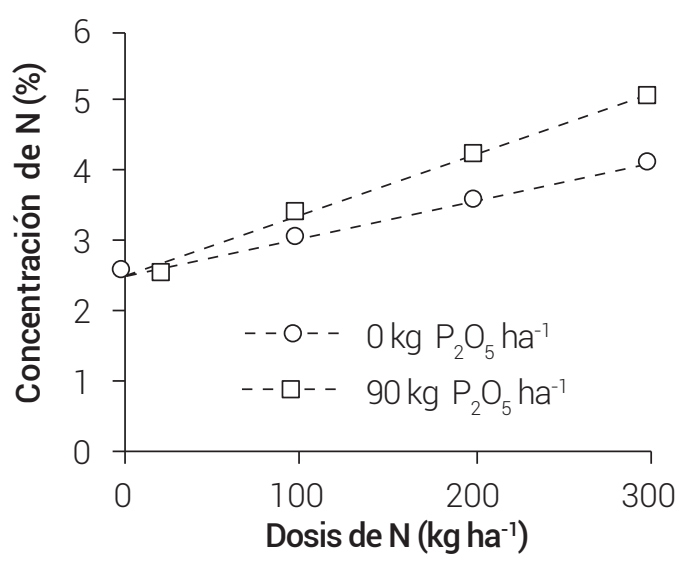

Figura 4. Concentraciones de nitrógeno en follaje de la verdolaga (Portulaca oleracea L.) en función del N y P aplicados.
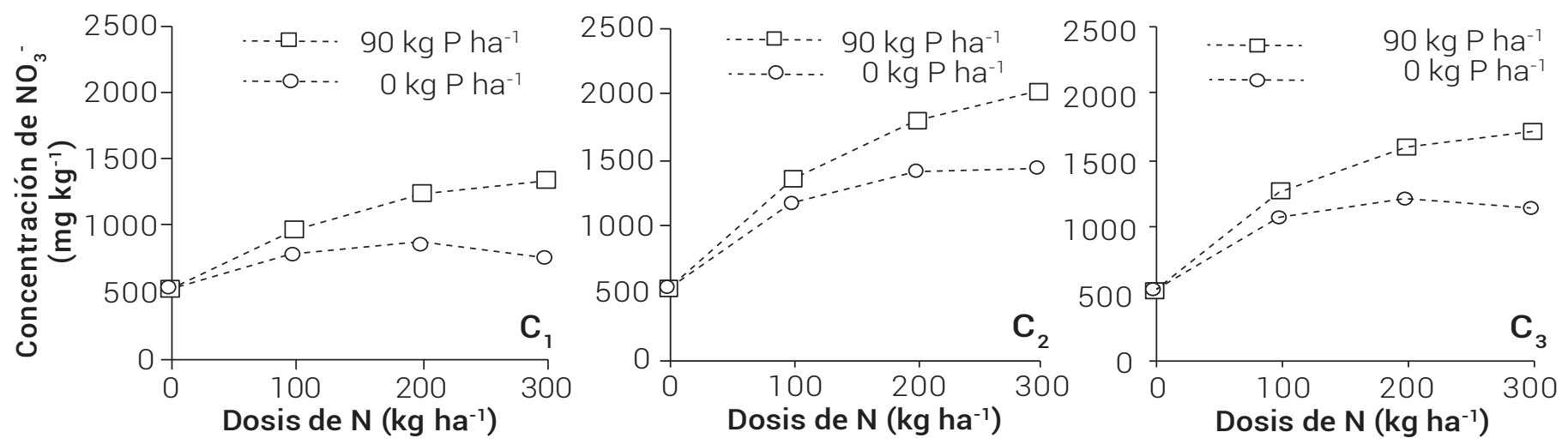

Figura 5. Concentración de $\mathrm{NO}_{3}^{-}$en el follaje de la verdolaga (Portulaca oleracea L.) en función del $\mathrm{N}$ y $\mathrm{P}$ aplicados, y fecha de cosecha. $C_{1}$ : cosecha 1 a 27 dde; $C_{2}$ : cosecha 2 a 34 dde y $C_{3}$ : cosecha 3 a 42 dde.

$\mathrm{ha}^{-1}$, para las tres cosechas, respectivamente (Figura 5). El efecto de $\mathrm{P}$ puede ser debido a que la mayor presencia de este elemento en el suelo favorece la mineralización de la materia orgánica y la nitrificación del amonio, lo cual se asocia a una mayor absorción de $\mathrm{NO}_{3}-$ por la planta (Havlin et al., 2005).

Las condiciones de cultivo influirán en la concentración de nitratos, pues al usar sulfato de amonio se obtiene la menor concentración de nitrato (1232 $\mathrm{mg} \mathrm{NO}_{3}{ }^{-} \mathrm{kg}^{-1} \mathrm{MF}$ ) (Kaymak, 2013), mientras que en condiciones salinas y con menor incidencia de luz se incrementan (de 1000 a $3000 \mathrm{mg} \mathrm{NO}_{3}{ }^{-} \mathrm{kg}^{-1} \mathrm{MF}$ ) (Franco et al., 2011). Por otra parte, las mayores concentraciones de nitrato se han reportado en sistemas hidropónicos, con 2520 y $3949 \mathrm{mg} \mathrm{NO}_{3}{ }^{-} \mathrm{kg}^{-1}$ MF (Egea-Gilabert et al., 2014; Kaşkar et al., 2009).

La concentración de $\mathrm{NO}_{3}^{-}$en verdolaga en este estudio es considerada de media a alta $\left(<2500 \mathrm{mg} \mathrm{NO}_{3}{ }^{-} \mathrm{kg}^{-1} \mathrm{MF}\right)$, con respecto a la clasificación de Santamaria (2006), aunque la Autoridad Europea de Seguridad Alimentaria (EFSA,
2008) menciona límites recomendados para la ingesta diaria de $3.7 \mathrm{mg} \mathrm{NO}_{3}{ }^{-} \mathrm{kg}^{-1}$ de peso corporal por día. Posteriormente, la Comisión de Regulación de la Unión Europea (2011) emitió el reglamento (UE) No 1258/2011 donde aumentó el umbral permitido para algunas hortalizas (3000 $\mathrm{mg} \mathrm{NO}_{3}{ }^{-} \mathrm{kg}^{-1} \mathrm{MF}$ ), teniendo en cuenta los posibles efectos positivos de la actividad antioxidante $u$ otras propiedades saludables en hortalizas; por lo tanto, la concentración de $\mathrm{NO}_{3}{ }^{-}$reportadas en este estudio no es problema para el consumo de la verdolaga en fresco.

\section{Dosis óptima económica}

El modelo de regresión de rendimiento de follaje indicó efecto positivo de la cosecha, la densidad de plantas y el $\mathrm{N}$ aplicado; al no observar interacción entre las cosechas y el $\mathrm{N}$ aplicado, la dosis óptima económica de $\mathrm{N}$ es la misma para las tres fechas de cosecha; en cambio, el rendimiento óptimo económico es mayor para las cosechas más tardías. La densidad de plantas tuvo un efecto lineal, por lo que los rendimientos óptimos económicos se obtuvieron 


\section{a 2500 plantas $\mathrm{m}^{-2}$.}

En el Cuadro 1 se presenta el análisis económico para el rendimiento de follaje de verdolaga en las tres fechas de cosecha, que comprende las dosis óptimas económicas y los costos variables para el $\mathrm{N}$ y densidad de plantas, el ingreso neto, el rendimiento óptimo económico y la relación beneficio costo.

Cabe señalar que el mayor costo de producción de la verdolaga se deriva de la semilla, estimado en 7950 pesos por hectárea, lo cual corresponde a $25 \mathrm{~kg}$ de semilla ha-1, debido a la baja germinación de la misma (de 12 a $28 \%$ ). La dosis óptima económica (DOE) se estimó en $65 \mathrm{~kg} \mathrm{~N}$ ha $^{-1}$ con 2500 plantas $\mathrm{m}^{-2}$, con rendimientos de follaje óptimos económicos de 58.83, 95.95 y $130.22 \mathrm{t} \mathrm{ha}^{-1}$, e ingresos netos de $30,338,67,828$ y 102,440 pesos mexicanos por hectárea, en las tres cosechas, respectivamente.

\section{CONCLUSIONES}

El rendimiento de follaje de la verdolaga fue afectado sólo por el $\mathrm{N}$ aplicado, la fecha de cosecha y la densidad de plantas $\mathrm{m}^{-2}$. Los máximos rendimientos de follaje se obtienen con la dosis más alta de $\mathrm{N}$ en las tres cosechas; sin embargo, el mayor incremento se obtuvo con $100 \mathrm{~kg}$ $\mathrm{N} \mathrm{ha}^{-1}$. La altura se incrementó por el $\mathrm{N}$ aplicado y la fecha de cosecha. La concentración de $\mathrm{Ny} \mathrm{NO}_{3}{ }^{-}$en el follaje de la verdolaga se incrementó por el $\mathrm{N}$ y $\mathrm{P}$ aplicados. Los contenidos de $\mathrm{NO}_{3}{ }^{-}$en el follaje son inferiores a los límites permitidos para su consumo en fresco. La dosis óptima económica de $\mathrm{N}$ y densidad de plantas en las tres fechas

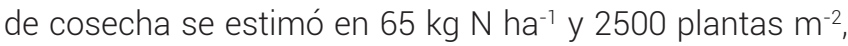
donde se obtiene el mayor rendimiento óptimo económico y el mayor ingreso neto.

\section{BIBLIOGRAFÍA}

Alcántar G. G. y M. Sandoval V. (1999) Manual de Análisis Químico de Tejido Vegetal. Publicación Especial No. 10. Sociedad Mexicana de la Ciencia del Suelo A. C. Chapingo, México. 156 p.

Bouyoucos G. J. (1936) Directions for making mechanical analysis of soils by the hydrometer method. Soil Science 42:225-230.

Brumfiel E. M. (2009) El estudio de la clase común: el asentamiento de Xaltocan durante el Posclásico en la cuenca de México. Cuiculco 47:59-86.

Byrne R. and J. H. McAndrews (1975) Pre-Columbian purslane (Portulaca oleracea L.) in the New World. Nature 253:726-727.

Chapman H. D. (1965) Cation exchange capacity. In: Methods of Soil Analysis. Part 2. Chemical and Microbiological Properties. Agronomy 9. C. A. Black, D. D. Evans, J. L. White, L. E. Ensminger and F. E. Clark (eds.). American Society of Agronomy. Madison WI, USA. pp:891-901

Chapman J., R. B. Stewart and R. A. Yarnell (1973) Archaeological evidence for precolumbian introduction of Portulaca oleracea and Mollugo verticillata into Eastern North America. Economic Botany 28:411-412.

Coltman R. R. (1989) Managing nitrogen fertilization of tomatoes using nitrate quick test. In: Tomato and Pepper Production in the Tropics. S. K. Green, T. D. Griggs and B. T. McLean (eds.). Asian Vegetable Research and Development Center (AVRDC). Shanhua, Tainan, Taiwan. pp:375-383.

Comisión de Regulación de la Unión Europea (2011) Reglamento (UE) No. 1258/2011 de la Comisión de 2 de diciembre de 2011 que modifica el Reglamento (CE) no 1881/2006 por lo que respecta al contenido máximo de nitratos en los productos alimenticios. Diario Oficial de la Unión Europea 3-12-2011. pp:320/15$320 / 17$.

Cros V., J. J. Martínez-Sánchez and J. A. Franco (2007) Good yields of common purslane with a high fatty acid content can be obtained in a peat-based floating system. HortTechnology 17:14-20.

Dibble C. E. and A. J. O. Anderson (1963) Florentine Codex: General History of the Things of New Spain. Book 11 - Earthly Things. Fray Bernardino de Sahagun. Translated by Dibble Charles E. and Anderson Arthur J. O. School of American Research and University of Utah. Monographs of the School of American Research and the Museum of New Mexico, No. 14, Pt. 12, University of Utah Press, Salt Lake City. pp:297.

Egea-Gilabert C., M. V. Ruiz-Hernández, M. A. Parra and J. A. Fernández (2014) Characterization of purslane (Portulaca oleracea L.) accessions: suitability as ready-to-eat product. Scientia Horticulturae 172:73-81

Elmi A. A., T. Mebrahtu, T. R. Omara-Alwala and M. Ezekwe (1997) Environmental effects on yield and agronomic traits of purslane (Portulaca spp.). Virginia Journal of Science 48:203-209.

Cuadro 1. Análisis económico de rendimiento de la verdolaga (Portulaca oleracea L.) en tres fechas de cosecha.

\begin{tabular}{|c|c|c|c|c|c|c|c|c|c|}
\hline \multirow{2}{*}{ Cosecha } & \multicolumn{2}{|c|}{ DOE } & \multirow{2}{*}{$\begin{array}{c}\text { Rendimiento } \\
\left(\mathrm{t} \mathrm{ha}^{-1}\right)\end{array}$} & IT & CVN & CVDP & $\mathrm{CT}$ & IN & \multirow[t]{2}{*}{$\mathrm{RBC}$} \\
\hline & $\left(\mathrm{kg} \mathrm{N} \mathrm{ha}^{-1}\right)$ & DP & & \multicolumn{5}{|c|}{$(\$$ mexicanos ha-1) } & \\
\hline \multirow{2}{*}{$\mathrm{C}_{1}$} & 0 & 2500 & 51.38 & 51,892 & 0 & 7950 & 25,280 & 26,612 & 1.05 \\
\hline & 65 & 2500 & 58.83 & 59,424 & 1904 & 7950 & 29,086 & 30,338 & 1.04 \\
\hline \multirow{2}{*}{$\mathrm{C}_{2}$} & 0 & 2500 & 88.49 & 89,382 & 0 & 7950 & 25,280 & 64,102 & 2.53 \\
\hline & 65 & 2500 & 95.95 & 96,915 & 1904 & 7950 & 29,086 & 67,828 & 2.33 \\
\hline \multirow{2}{*}{$\mathrm{C}_{3}$} & 0 & 2500 & 122.77 & 123,994 & 0 & 7950 & 25,280 & 98,714 & 3.90 \\
\hline & 65 & 2500 & 130.22 & 131,526 & 1904 & 7950 & 29,086 & 102,440 & 3.52 \\
\hline
\end{tabular}

DOE: dosis óptima económica; DP. densidad de plantas (plantas $\mathrm{m}^{-2}$ ); IT: ingreso total ( $\$$ ha $\left.^{-1}\right)$, CVN: costo variable de nitrógeno (\$ ha-1); CVDP. costo variable de densidad de plantas $\left(\$ \mathrm{ha}^{-1}\right)$; CT: costo total $\left(\$ \mathrm{ha}^{-1}\right)$; IN: ingreso neto (\$ ha-1), y RBC: relación beneficio costo; $\mathrm{C}_{1}$ : cosecha 1 (27 dde); $\mathrm{C}_{2}$ : cosecha 2 (34 dde); $\mathrm{C}_{3}$ : cosecha 3 (42 dde). 
EFSA, European Food Safety Authority (2008) Nitrate in vegetables. Scientific opinion of the panel on contaminants in the food chain. The EFSA Journal 689:1-79.

Ezekewe M. O., T. R. Omara-Alwala and T. Membrahtu (1999) Nutritive characterization of purslane accessions as influenced by planting date. Plant Foods for Human Nutrition 54:183-191.

Fontana E., J. Hoeberechts, S. Nicola, V. Cros, G. B. Palmegiano and P. G. Peiretti (2006) Nitrogen concentration and nitrate/ammonium ratio affect yield and change the oxalic acid concentration and fatty acid profile of purslane (Portulaca oleracea L.) grown in a soilless culture system. Journal of the Science of Food and Agriculture 86:2417-2424.

Franco J. A., V. Cros, M. J. Vicente and J. J. Martínez-Sánchez (2011) Effects of salinity on the germination, growth, and nitrate contents of purslane (Portulaca oleracea L.) cultivated under different climatic conditions. The Journal of Horticultural Science and Biotechnology 86:1-6.

García E. (1973) Modificaciones al Sistema de Clasificación Climática de Köppen. Instituto de Geografía. Universidad Nacional Autónoma de México. México, D.F. 246 p.

Graifenberg A., L. Botrini, L. Giustiniani, F. Filippi and M. Curadi (2003) Tomato growing in saline conditions with biodesalinating plants: Salsola soda L., and Portulaca oleracea L. Acta Horticulturae 609:301-305.

Havlin J. L., J. D. Beaton, S. L. Tisdale and W. L. Nelson (2005) Soil Fertility and Fertilizers: an Introduction to Nutrient Management. Pearson Prentice Hall. Upper Saddle River, New Jersey, USA. 515 p.

Jackson M. L. (1976) Análisis Químico de Suelos. J. B. Martínez (trad.). Omega. Barcelona, España. 662 p.

Kaşkar C., J. A. Fernández, J. Ochoa, D. Niñirola, E. Conesa and Y. Tüzel (2009) Agronomic behaviour and oxalate and nitrate content of different purslane cultivars (Portulaca oleracea) grown in a hydroponic floating system. Acta Horticulturae 807:521-526.

Kaymak H. C. (2013) Effect of nitrogen forms on growth, yield and nitrate accumulation of cultivated purslane (Portulaca oleracea L.). Bulgarian Journal of Agricultural Science 19:444-449.

Kiliç C. C., Y. S. Kukul and D. Anaç (2008) Performance of purslane (Portulaca oleracea L.) as a salt-removing crop. Agricultural Water Management 95:854-858.

Liu L., P. Howe, Y. F. Zhou, Z. Q. Xu, C. Hocart and R. Zhang (2000) Fatty acids and $\beta$-carotene in Australian purslane (Portulaca oleracea) varieties. Journal of Chromatography A 893:207-213.

López R. G. F. (1996) Domesticación de la verdolaga (Portulaca oleracea L.) y semidomesticación del romerito (Suaeda diffusa Wats.) en el sistema agrícola de chinampas de San Gregorio Atlapulco, Xochimilco, D.F. Revista Geografía Agrícola 22-23:103-112.

McClung de Tapia E., D. Martínez Y., E. Ibarra M. and C. C. Adriano M. (2014)
Los orígenes prehispánicos de una tradición alimentaria en la Cuenca de México. Anales de Antropología 48:97-121.

Mohamed A. I. and A. S. Hussein (1994) Chemical composition of purslane (Portulaca oleracea). Plant Foods for Human Nutrition 45:1-9.

Olsen S. R. and L. A. Dean (1965) Phosphorus. In: Methods of Soil Analysis. Part 2. Chemical and Microbiological Properties. Agronomy 9. C. A. Black, D. D. Evans, J. L. White, L. E. Ensminger and F. E. Clark (eds.). American Society of Agronomy. Madison, WI, USA pp:1035-1049.

Palaniswamy U. R., R. J. McAvoy and B. B. Bible (2000) Omega-3-fatty acid concentration in Portulaca oleracea is altered by nitrogen source in hydroponic solution. Journal of the American Society for Horticultural Science 125:190-194.

Petropoulos S. A., A. Karkanis, A. Fernandes, L. Barros, I. C. F. R. Ferreira, G. Ntatsi, K. Petrotos, C. Lykas and E. Khah (2015) Chemical composition and yield of six genotypes of common purslane (Portulaca oleracea L.): an alternative source of omega-3 fatty acids. Plant Foods for Human Nutrition 70:420-426.

Richards L. A. (1990) Diagnóstico y Rehabilitación de Suelos Salinos y Sódicos. Departamento de Agricultura de Estados Unidos de América. Limusa. México, D.F. 172 p.

Santamaria P. (2006) Nitrate in vegetables: toxicity, content, intake and EC regulation. Journal of the Science of Food and Agriculture 86:10-17.

Siriamornpun S. and M. Suttajit (2010) Microchemical components and antioxidant activity of different morphological parts of thai wild purslane (Portulaca oleracea). Weed Science 58:182-188.

Soil Survey Staff (2010) Claves para la Taxonomía de Suelos. 11a ed. C. A. Ortiz-Solorio and M. C. Gutiérrez-Castorena (Trads.). USDANatural Resources Conservation Service, Washington, D.C. USA.

Teixeira M. and I. S. Carvalho I. (2009) Effects of salt stress on purslane (Portulaca oleracea) nutrition. Annals of Applied Biology 154:77-86.

Uddin M. K., A. S. Juraimi, F. Anwar, M. A. Hossain and M. A. Alam (2012) Effect of salinity on proximate mineral composition of purslane (Portulca oleracea L.). Australian Journal of Crop Science 6:1732-1736.

Vázquez-Alonso M. T., R. Bye, L. López-Mata, M. T. P. Pulido-Salas, E. McClung de T. y S. D. Koch (2014) Etnobotánica de la cultura teotihuacana. Botanical Science 92:563-574.

Volke H. V. (1982) Optimización de Insumos de la Producción en la Agricultura. Colegio de Postgraduados. Montecillo, Estado de México. $61 \mathrm{p}$

Volke H. V. (2008) Estimación de Funciones de Respuesta para Información de Tipo no Experimental, Mediante Regresión. Colegio de Postgraduados. Montecillo, Estado de México. 113 p. 